\title{
Leptin and the GA genotype of rs2167270 of the $L E P$ gene increase the risk of prediabetes
}

\author{
MUKHALLAD A. ALJANABI $^{1}$, MAHMOUD A. ALFAQIH ${ }^{1}$, MARIAM KHANFAR ${ }^{1}$, \\ ZOUHAIR O. AMARIN ${ }^{2}$, LINA ELSALEM ${ }^{3}$, RAMI SAADEH ${ }^{4}$ and FAHEEM AL-MUGHALES ${ }^{1}$ \\ Departments of ${ }^{1}$ Physiology and Biochemistry, ${ }^{2}$ Obstetrics and Gynecology, \\ ${ }^{3}$ Pharmacology and ${ }^{4}$ Community Medicine and Public Health, \\ School of Medicine, Jordan University of Science and Technology, Irbid 22110, Jordan
}

Received July 21, 2020; Accepted February 2, 2021

DOI: $10.3892 /$ br.2021.1420

\begin{abstract}
Prediabetes is a precursor stage that frequently develops to definitive type 2 diabetes mellitus (T2DM). Therefore, identifying individuals with prediabetes can allow for early intervention measures that delay or prevent disease progression to T2DM. Several biochemical changes appear to be associated with prediabetes, including an increase in the serum levels of leptin. In Jordan, this association has not been previously investigated. In the present study, the serum levels of leptin were measured in 122 prediabetes subjects and 122 controls. Furthermore, the genotypes of three single nucleotide polymorphisms in the LEP gene (rs7799039, rs2167270 and rs791620) were investigated for their association with prediabetes using PCR-restriction fragment length polymorphism. The results revealed a significant increase in serum leptin levels in the prediabetes group. It was also shown that the GA genotype and the A allele of rs2167270 were significantly associated with an increased risk of prediabetes $(\mathrm{P}<0.05)$. These findings were shown to be independent of body mass index, waist circumference and serum glucose levels. To the best of our knowledge, the present study is the first in Jordan to have reported an association between serum leptin levels and the GA genotype of rs 2167270 with an increased risk of prediabetes, identified both in the univariate and multivariate models.
\end{abstract}

Correspondence to: Dr Mahmoud A. Alfaqih, Department of Physiology and Biochemistry, School of Medicine, Jordan University of Science and Technology, P.O. Box 3030, M2-L0, Irbid 22110, Jordan E-mail: maalfaqih@just.edu.jo

Abbreviations: T2DM, type 2 diabetes mellitus; SNP, single nucleotide polymorphism; IDF, international diabetes federation; KAUH, King Abdullah University Hospital; WC, waist circumference; TG, triglycerides; RFLP, restriction fragment length polymorphism; HWE, Hardy-Weinberg equilibrium; LR, leptin resistance; HOMA-IR, homeostatic model assessment of insulin resistance

Key words: T2DM, leptin, prediabetes, Jordan, LEP gene, insulin resistance, SNP

\section{Introduction}

Type 2 diabetes mellitus (T2DM) is one of the most prevalent and challenging chronic illnesses of the $21^{\text {st }}$ century (1). It is characterized by chronic hyperglycemia and impaired metabolism of carbohydrates, fats and protein (2). T2DM is primarily caused by $\beta$-cell dysfunction accompanied by increased insulin resistance in the target tissue (3). In terms of the short- and long-term effects of T2DM, it is ranked as the $7^{\text {th }}$ major cause of mortality worldwide according to the World Health Organization (4). Globally, the International Diabetes Federation (IDF) have reported that $\sim 10 \%$ of the worlds population have T2DM. Indeed, 415 million individuals were reported to have T2DM in 2015, a number predicted to reach 642 million by 2040 (5). A sedentary lifestyle, together with increased consumption of unhealthy diets, are major triggers for the development of T2DM (1).

Previous estimates of the prevalence of T2DM in the Middle East and North Africa (MENA) region ranks it as the second highest worldwide. If current trends continue, the number of individuals with T2DM is expected to increase by $96.2 \%$ by 2035 (6). Statistics from the IDF indicate that Jordan, a country in the MENA region, ranks $26^{\text {th }}$ in the prevalence of T2DM (5). A study of T2DM trends in Jordan over a 23-year period reported a $10.7 \%$ increase in the prevalence of T2DM between 1994 and $2017(7,8)$.

Our understanding of the progression of T2DM highlights the presence of a transitional stage between normal blood glucose levels in healthy individuals and the characteristic hyperglycemia of T2DM. During this stage, commonly referred to as intermediate hyperglycemia or prediabetes, individuals develop insulin resistance, which results in an elevated level of blood glucose, albeit still lower than the reference values of T2DM (9).

The American Diabetes Association estimates that $70 \%$ of all individuals with prediabetes will eventually transition into T2DM (10). However, a growing body of evidence accumulated over the last few decades has indicated that lifestyle changes, such as a healthier diet and exercise, may decelerate or even halt this progression $(11,12)$. Evidence also suggests that pharmacological intervention using therapeutics that modify insulin resistance may also supplement/replace the need for 
the above non-pharmacological interventions, and may also delay or prevent progression to T2DM (13).

Given the above, it is imperative to diagnose individuals with prediabetes, and to introduce the above intervention strategies, whether pharmacological or non-pharmacological, at an early stage before progression into T2DM. One approach to help in the diagnosis of prediabetic individuals is to investigate the biochemical changes that are associated with an increased risk of prediabetes. The identification of the above changes would not only help with improved diagnosis, but it may also provide an improved understanding of the pathogenesis of the disease and aid the monitoring of disease progression.

Leptin is an adipokine hormone secreted primarily by adipocytes (14). Leptin regulates body fat through the suppression of appetite, induction of energy expenditure and regulation of glucose homeostasis $(15,16)$. Inactivating mutations in the gene that encodes leptin $(L E P)$ were found to be associated with obesity in animal models, and $L E P$ was initially identified as the 'obese gene' (17). Counterintuitively, an elevation in the levels of serum leptin in obese individuals was observed in several cohorts (18-22). This led to the proposition that resistance to leptin, rather than its absolute levels, is a major characteristic of obesity in humans $(23,24)$. This is supported by evidence showing that insulin, levels of which are often increased in obese individuals, stimulates leptin secretion (16).

Individuals with prediabetes are commonly obese, and have elevated levels of serum insulin to compensate for resistance to insulin action. Not surprisingly, data from several cohorts have demonstrated an elevation in serum leptin levels in these individuals $(24,25)$. This observation however, was not consistent across all the different study cohorts, and was not tested in individuals from Jordan (26). The risk of developing prediabetes is multifactorial; in addition to the role exerted by environmental factors, it may be affected by genetic variations $(9,18)$. Interestingly, $L E P$ variants were reported to be strongly associated with obesity and insulin resistance (19,27-29). In the present study, the association between serum leptin and the following single nucleotide polymorphisms (SNPs), rs7799039, rs2167270 and rs791620, were examined in LEP with respect to assessing the risk of prediabetes in patients in Jordan.

\section{Materials and methods}

Patients. The present study was a case-controlled study involving 122 individuals with prediabetes and 122 healthy controls. In the prediabetes group, 67 of the recruited participants were females, with an age range of 34-66 years and a median age of 49 years, and 55 males, with an age range of 40-68 years and a median age of 51 years. In the control group, 67 of the recruited participants were females with an age range of 32-76 years and a median age of 49, as well as 55 males with an age range of 36-72 years and a median age of 54 years. Prior to subject recruitment, the sample size required to show statistically significant differences in serum leptin in a case control design was calculated using Epitool (epitools.ausvet. com.au/samplesize). It was determined that a sample size of 98 subjects in each group was sufficient to conduct a study that has $80 \%$ statistical power, $5 \%$ margin of error, $95 \%$ confidence interval (CI), and an assumed odds ratio (OR) of 4. The study was approved by the Institutional Review Board of Jordan University of Science and Technology and its affiliated teaching hospital [King Abdullah University Hospital (KAUH); approval no. 90/118/2018]. Subjects were recruited from the Family Medicine clinics of KAUH. The demographic data, anthropometric measurements [height, weight and waist circumference (WC)] and medical history, along with patients' written consents and signatures, were collected from all subjects upon an interview with a clinical research coordinator. Healthy controls and prediabetes subjects were matched according to their sex and age.

Collection of blood and serum samples. From each patient, two blood samples ( $5 \mathrm{ml}$ each) were collected following a $15 \mathrm{~h}$ overnight fast. One sample was collected in an EDTA tube (AFCO-Jordan) and stored at $4^{\circ} \mathrm{C}$ for use in DNA extraction; the other sample was collected in a plain tube with gel clot activator (AFCO-Jordan) and used to obtain serum following centrifugation at room temperature for $5 \mathrm{~min}$ at 4,000 $\mathrm{x} \mathrm{g}$.

The collected serum was subsequently used to assess the levels of leptin, fasting glucose, total cholesterol and triglycerides (TGs). Subjects with fasting serum glucose levels of $100-125 \mathrm{mg} / \mathrm{dl}$ were requested to repeat the test in the following month, and were considered to have prediabetes if their fasting serum glucose levels remained within this range. Subjects with fasting serum glucose levels $<100 \mathrm{mg} / \mathrm{dl}$ were assigned to the control group. All individuals with serum glucose levels $\geq 126 \mathrm{mg} / \mathrm{dl}$ were excluded from the study, and were referred to the Endocrinology clinic at KAUH to confirm a tentative diagnosis of T2DM.

Biochemical measurements. The levels of serum glucose, total cholesterol and TG were measured using a Roche automated clinical analyzer system (Roche Diagnostics $\mathrm{GmbH}$ ). A sandwich ELISA was used to assess serum leptin levels using a human ELISA kit (cat. no. MBS020274; MyBioSource, Inc.). The minimum detection level was $10 \mathrm{pg} / \mathrm{ml}$, and the protocol used is described by Saadeh et al (30).

DNA extraction and genotyping. Genomic DNA was extracted from the collected blood in EDTA tubes using a QIAamp DNA Blood Mini kit (Qiagen $\mathrm{GmbH}$ ) according to the protocol described by Alfaqih et al (31). The DNA concentration was measured spectrophotometrically at $260 \mathrm{~nm}$ using an ND-2000 Nanodrop spectrophotometer (Thermo Fisher Scientific, Inc.). DNA purity was assessed using the same spectrophotometer by calculating the $260 / 280 \mathrm{~nm}$ ratio.

The genotypes of the three SNPs of the LEP gene (rs7799039, rs2167270 and rs791620) were examined using the PCR-restriction fragment length polymorphism (PCR-RFLP) technique. SNP information was obtained from the NCBI dbSNP database (ncbi.nlm.nih.gov/snp/rs7799039, ncbi.nlm. nih.gov/snp/rs2167270 and ncbi.nlm.nih.gov/snp/rs791620). Each PCR reaction was composed of GoTaq ${ }^{\circledR}$ Green MasterMix (Promega Corporation), $0.4 \mu \mathrm{M}$ primers (forward and reverse) and $5 \mathrm{ng}$ sample DNA, bringing the total reaction volume to $25 \mu \mathrm{l}$. The PCR thermocycling conditions, as well as the sequences of the forward and reverse primers of each SNP are shown in Table I.

The PCR-RFLP assay details, including the location of the SNPs on the LEP gene, and the size of the PCR amplicon, 


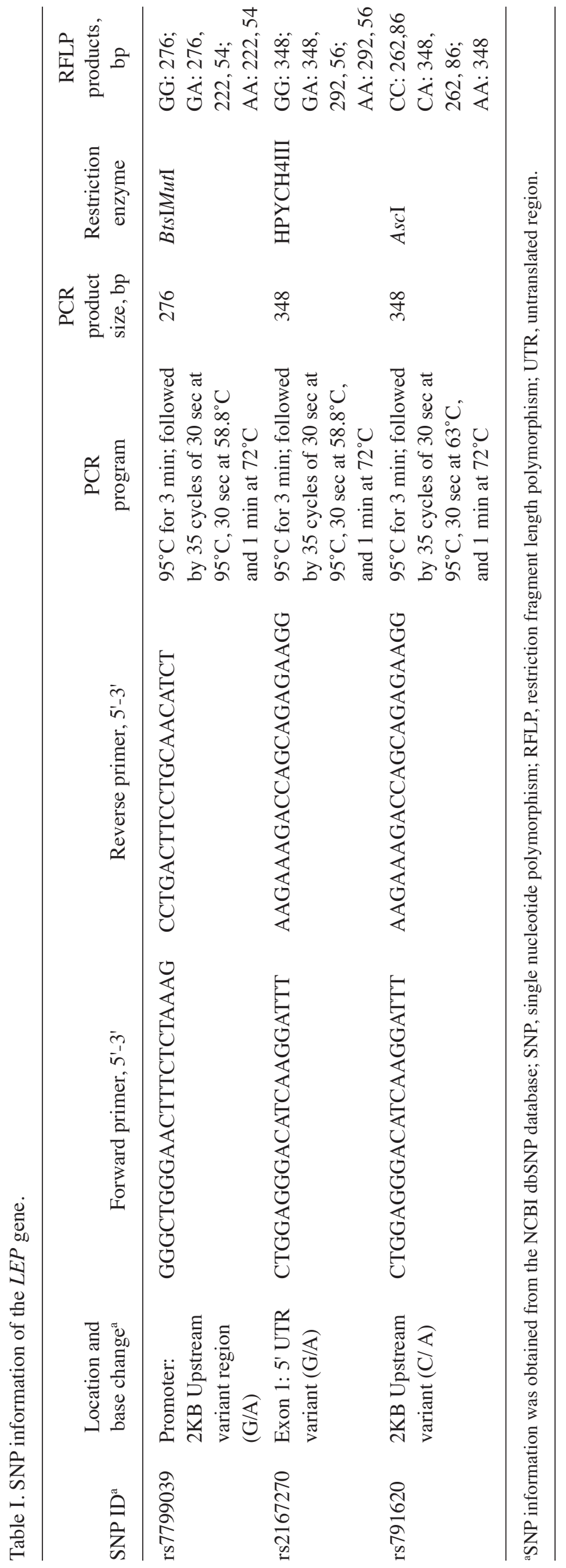


Table II. Baseline variable of the subjects.

\begin{tabular}{llcc}
\hline Variable & Control, $\mathrm{n}=122^{\mathrm{c}}$ & Prediabetes, $\mathrm{n}=122^{\mathrm{c}}$ & P-value \\
\hline Sex & & & 1 \\
Males & $55(45 \%)$ & $55(45 \%)$ & \\
Females & $67(55 \%)$ & $67(55 \%)$ & 0.13 \\
Age, years & $51.50 \pm 9.35$ & $49.80 \pm 8.24$ & $0.02^{\mathrm{a}}$ \\
BMI, kg/m & $30.70 \pm 6.00$ & $32.40 \pm 6.19$ & $<0.0001^{\mathrm{b}}$ \\
Waist circumference, cm & $103.6 \pm 3.0$ & $110.5 \pm 14.1$ & $<0.0001^{\mathrm{b}}$ \\
Glucose, $\mathrm{mg} / \mathrm{dl}$ & $90.00 \pm 13.5$ & $118.8 \pm 20.2$ & 0.08 \\
Cholesterol, mg/dl & $185.7 \pm 43.7$ & $195.9 \pm 49.1$ & 0.95 \\
Triglycerides, mg/dl & $165.2 \pm 124$ & $164.4 \pm 93.1$ & $<0.0001^{\mathrm{b}}$ \\
Leptin, ng/dl & $27.50 \pm 22.2$ & $49.60 \pm 54.9$ & $<0.0001^{\mathrm{b}}$ \\
Leptin/BMI & $0.867 \pm 0.620$ & $1.487 \pm 1.58$ & \\
\hline
\end{tabular}

${ }^{\mathrm{a}} \mathrm{P}<0.05,{ }^{\mathrm{b}} \mathrm{P}<0.0001$. $^{\mathrm{c} D a t a}$ are presented as the mean \pm standard deviation, or $\mathrm{n}(\%)$. BMI, body mass index.

are summarized in Table I. The PCR product of each SNP was later digested using the appropriate restriction enzymes. The restriction enzymes used for genotyping, and the size of the products of each genotype following digestion with the corresponding restriction enzyme are listed in Table I. The undigested PCR products and the DNA fragments resulting from the restriction enzyme digestion were separated on a $3 \%$ agarose gel stained with $0.5 \mu \mathrm{g} / \mathrm{ml}$ ethidium bromide and subsequently visualized under ultraviolet light. The genotype and allele call rates of all the investigated SNPs were 100\%.

Statistical analysis. Statistical analysis was performed using SPSS version 23 (IBM Corp.). The statistical differences between the prediabetes and control groups in terms of the levels of serum glucose, total cholesterol, TGs, leptin, body mass index (BMI), sex, age and WC were assessed using a Student's t-test. A Pearson's $\chi^{2}$ test was used assess the differences in sex distribution, whereas a Fischer's exact test was used to examine the association between genotype or allele categories with the risk of prediabetes. The above test was also used to test whether the three SNPs were conformant with the Hardy-Weinberg equilibrium (HWE). Haplotype association analysis was performed using the SHEsis online platform (analysis.bio-x.cn/myAnalysis.php).

Multivariate logistic regression analysis was used to determine whether age, sex, serum leptin, rs7799039, rs2167270 or rs791620 were statistically associated with prediabetes following adjustments for serum glucose, BMI and WC. The Forward Wald statistics methods was used for variable entry. $\mathrm{P}<0.05$ and the $95 \%$ confidence interval (CI) were considered to indicate a statistically significant difference.

\section{Results}

Patient characteristics and biochemical profile. A total of 122 healthy subjects and 122 subjects with prediabetes were recruited for the present study. The subjects were matched for sex and age. Females comprised 55\% of the total subjects in each group (Table II). Individuals with prediabetes had significantly higher BMI $(\mathrm{P}=0.02)$ and $\mathrm{WC}(\mathrm{P}<0.0001)$ values compared with the control group. Biochemical analysis of the subjects in each group showed that the subjects with prediabetes had significantly higher levels of serum glucose and leptin, and a greater leptin/BMI ratio $(\mathrm{P}<0.0001$; Table II). Total serum cholesterol or serum TG levels were not significantly different between the two groups (Table II).

LEP genotyping. Subjects in the control and prediabetes groups were genotyped for three SNPs in the LEP gene (rs7799039, rs2167270 and rs791620). The genotyping results of all three SNPs did not deviate from the HWE. The genotype and allele frequencies of the above SNPs are shown in Tables III and IV, respectively. This analysis revealed significant differences in the genotype and allele frequencies of rs 2167270 . For this variant, the prediabetes group had a significantly higher frequency of the heterozygous GA genotype compared with the control group (63 cf. 35\%; Table III). Having the GA genotype of rs 2167270 increased the risk of prediabetes by $\sim 3$-fold [odds ratio $(\mathrm{OR})=3.1 ; \mathrm{CI}=1.8-5.6 ; \mathrm{P}<0.0001]$. The frequency of the A allele of rs2167270 was significantly higher in subjects with prediabetes compared with the control group (37 cf. 27\%; $\mathrm{P}=0.03$; Table IV).

In order to identify a chromosomal block in the LEP gene associated with the risk of prediabetes, the associations of different haplotypes resulting from genotyping all three SNPs of the $L E P$ gene were tested. The results of the above analysis are shown in Table V. The GGC haplotype of rs 2167270 , rs7799039 and rs791620, which contains the major allele of each SNP, had a lower frequency in the prediabetes group compared with controls, and was associated with a significantly lower risk of prediabetes $(\mathrm{OR}=0.47, \mathrm{CI}=0.335-0.655$; $\mathrm{P}<0.0001)$. Moreover, the AGC haplotype, which contains the high-risk A allele of rs2167270, was associated with a significantly increased risk of prediabetes $(\mathrm{OR}=1.53, \mathrm{CI}=1.022-2.299$; $\mathrm{P}<0.0001)$.

Multivariate analysis. Considering that the prediabetes and the control groups were significantly different with respect to 
Table III. Genotype frequencies of the rs7799039, rs2167270 and rs791620 SNPs in control and subjects with prediabetes.

\begin{tabular}{lccccc}
\hline SNPID & Genotype & Control, $\mathrm{n}(\%)$ & Prediabetes, $\mathrm{n}(\%)$ & OR $(95 \% \mathrm{CI})$ & P-value \\
\hline rs7799039 & G/G & $49(40)$ & $33(27)$ & 1 & 0.09 \\
& G/A & $54(44)$ & $68(56)$ & $1.87(1.06-3.30)$ & \\
rs2167270 & $\mathrm{A} / \mathrm{A}$ & $19(16)$ & $21(17)$ & $1.64(0.77-3.51)$ & \\
& $\mathrm{G} / \mathrm{G}$ & $67(55)$ & $38(31)$ & 1 & $<0.0001$ \\
& $\mathrm{G} / \mathrm{A}$ & $43(35)$ & $77(63)$ & $3.16(1.83-5.45)$ & \\
rs791620 & $\mathrm{A} / \mathrm{A}$ & $12(10)$ & $7(6)$ & $1.03(0.37-2.83)$ & \\
& $\mathrm{C} / \mathrm{C}$ & $118(97)$ & $120(98)$ & 1 & 0.68 \\
& $\mathrm{C} / \mathrm{A}$ & $3(2)$ & $2(2)$ & $0.66(0.11-3.99)$ & \\
\hline
\end{tabular}

SNP, single nucleotide polymorphism; OR, odds ratio; CI, confidence interval.

Table IV. Allele frequencies of rs7799039, rs2167270, and rs791620 SNPs in control and prediabetic subjects.

\begin{tabular}{|c|c|c|c|c|}
\hline SNP ID & Allele & Control, n (\%) & Prediabetes, n (\%) & P-value \\
\hline \multirow[t]{2}{*}{ rs7799039 } & G & $152(62)$ & $134(55)$ & \multirow[t]{2}{*}{0.11} \\
\hline & A & $92(38)$ & $110(45)$ & \\
\hline \multirow[t]{2}{*}{ rs2167270 } & $\mathrm{G}$ & $177(73)$ & $153(63)$ & \multirow[t]{2}{*}{$0.03^{\mathrm{a}}$} \\
\hline & A & $67(27)$ & $91(37)$ & \\
\hline \multirow[t]{2}{*}{ rs791620 } & $\mathrm{C}$ & $239(98)$ & $242(99)$ & \multirow[t]{2}{*}{0.44} \\
\hline & A & $5 \quad(2)$ & $2(1)$ & \\
\hline
\end{tabular}

${ }^{\mathrm{a}} \mathrm{P}<0.05$. SNP, single nucleotide polymorphism.

their BMI, WC and serum glucose measurements, multiple logistic regression analysis was subsequently performed following adjustments for BMI, WC and glucose. The results of this analysis are shown in Table VI. The serum leptin levels remained a significant predictor of an increased risk of prediabetes $(\mathrm{OR}=1.022,95 \% \mathrm{CI}=1.002-1.042 ; \mathrm{P}=0.029)$. Furthermore, similar to the findings of the univariate analysis, the GA genotype of rs2167270 was associated with a significantly increased risk of prediabetes compared with the $\mathrm{GG}$ genotype $(\mathrm{OR}=3.826,95 \% \mathrm{CI}=1.543-9.546$; $\mathrm{P}=0.004)$. None of the other SNPs in $L E P$ were significantly associated with prediabetes in this model.

\section{Discussion}

Leptin, a hormone secreted primarily by adipocytes, have been positively associated with body fat as well as adipocyte number and size (14). Several studies have examined the association between leptin levels and obesity, insulin resistance, metabolic syndrome and diabetes $(24,32)$. In addition, several studies have explored the association between leptin levels in different populations with prediabetes, where certain studies identified an association between them, and others did not $(24,26)$.

In Jordan, a small number of studies have investigated correlations between serum leptin levels with obesity and the metabolic syndrome $(23,33)$. However, to the best of our knowledge, the association between serum leptin and the risk of prediabetes has not been explored before in Jordan. The results of the univariate and multivariate analyses presented in the current study, in addition to findings from other populations, indicate an association between serum leptin and an increased risk of prediabetes.

The above findings lend support to the tentative utility of serum leptin for the identification of individuals at a higher risk of prediabetes. They also highlight a potential role for leptin in mediating the pathophysiology of the disease. In addition to the aforementioned results describing the association between leptin protein and prediabetes, the present study also identified an association between prediabetes and the SNP rs2167270 in the $L E P$ gene, which encodes the leptin protein (28). This finding further strengthens the association between leptin protein and prediabetes described above. Furthermore, it also provides evidence that the risk of developing prediabetes is multifactorial, involving lifestyle, environmental and genetic factors $(11,25)$.

Leptin is a hormone that normally inhibits appetite and increases energy expenditure (15). The exact mechanism(s) by which leptin induces these effects have yet to be fully delineated, although they appear to be modulated through binding of leptin to its receptors in the hypothalamus, which acts as the central orchestrator of hunger and satiety signals $(34,35)$. It therefore appears counterintuitive that serum levels of leptin are actually elevated in prediabetic individuals who are commonly obese and/or suffer from insulin resistance $(16,22)$. However, the observations regarding the levels of leptin in prediabetes are in agreement with previous reports demonstrating elevated levels of leptin in obese subjects, and in individuals with metabolic syndrome $(32,33)$.

The uniformity of these observations in multiple diseases that share common features indicate that resistance to leptin activity, rather than its absolute levels in the serum, is a common process involved in the pathophysiology of obesity, metabolic syndrome and T2DM $(21,23)$. However, despite the plethora of evidence linking leptin resistance (LR) to a number of different metabolic disorders, this field suffers from the absence of a method for the accurate and reproducible 
Table V. Haplotype frequency of rs2167270, rs7799039, and rs791620 SNPs in control and prediabetic patients. rs2167270 rs7799039 rs791620 Controls frequency ${ }^{\mathrm{b}}$ Prediabetes frequency Odds ratio 95\% confidence interval P-value

\begin{tabular}{|c|c|c|c|c|c|c|c|}
\hline A & A & $\mathrm{C}$ & 0.043 & 0.062 & 1.45 & $0.643-3.252$ & 0.37 \\
\hline A & G & $\mathrm{C}$ & 0.225 & 0.311 & 1.53 & $1.022-2.299$ & 0.03 \\
\hline $\mathrm{G}$ & A & $\mathrm{C}$ & 0.328 & 0.389 & 1.28 & $0.884-1.861$ & 0.19 \\
\hline $\mathrm{G}$ & G & $\mathrm{C}$ & 0.383 & 0.230 & 0.47 & $0.315-0.696$ & $<0.001^{\mathrm{a}}$ \\
\hline
\end{tabular}

${ }^{\mathrm{a}}<0.001 .{ }^{\mathrm{b}} \mathrm{A}$ frequency $<0.03$ in both controls and prediabetes were excluded.

Table VI. Multivariate analysis of leptin and LEP single nucleotide polymorphisms with the risk of prediabetes.

\begin{tabular}{lccc}
\hline Variable & Odds ratio & Confidence interval & P-value \\
\hline Leptin & 1.022 & $1.002-1.042$ & 0.029 \\
rs2167270 & & & \\
GG & 1 & & \\
GA & 3.826 & $1.543-9.546$ & 0.004 \\
AA & 1.543 & $0.380-6.268$ & 0.544 \\
\hline
\end{tabular}

Variables in the model include age, sex, leptin levels, rs7799039, rs2167270, and rs791620. The model was adjusted for glucose, body mass index and waist circumference. Method of variables entry in the model: Forward Wald Statistics.

measurement of LR. The derivation of such a method may aid in monitoring the progression of several metabolic disorders, including the response of patients to the different regimens applied for the treatment of these diseases.

In addition to the effect leptin has on energy metabolism through binding to its hypothalamic receptors, it may also regulate energy expenditure through indirect effects on insulin activity. Denroche et al (15) suggested that increased leptin levels in the serum may predispose individuals to insulin resistance via the uncoupling of insulin binding to its receptor from downstream signaling pathways, an effect mediated through inhibition of insulin receptor autophosphorylation by leptin protein.

It is interesting to note that Mazahreh et al (23) recently reported that laparoscopic sleeve gastrectomy (LSG), a bariatric surgical procedure, in morbidly obese individuals reduces serum leptin levels as well as the BMI (23). Given the above observation and the findings of the present study, it would be interesting to assess whether LSG reverses prediabetes in morbidly obese patients, possibly through a mechanism that involves a reduction in serum leptin/LR.

Another major finding of the present study was the association of SNP rs2167270 in the LEP gene with prediabetes. Specifically, in the univariate and multivariate analyses, the GA heterozygous genotype of rs 2167270 was associated with an increased risk of prediabetes. Furthermore, the results obtained indicated that the minor A allele of rs2167270 was more frequently observed in patients with prediabetes. Along the same lines, a haplotype of all three SNPs examined in the present study that included the A allele of rs2167270 was found to increase the risk of prediabetes, whereas another haplotype that contained the major $\mathrm{G}$ allele was protective against the disease. Taken together, these data have indicated that the minor A allele of rs2167270 is a high-risk allele that may be involved in the pathophysiology of prediabetes. The above SNP is located in the 5'-untranslated region of LEP (36). The exact mechanism by which this SNP modifies the prediabetes risk is unknown, although it may involve an effect of this SNP on LEP mRNA stability. Using in vitro methods in relevant cell lines, or animal model studies may help to unravel the precise mechanism(s).

One limitation of the present study was that any index that reflects insulin resistance in the study subjects was not measured. An easy-to-measure index would be the homeostatic model assessment of insulin resistance (HOMA-IR) (37). Measurement of HOMA-IR in the study subjects would have enabled the research team to have investigated whether the association of higher serum leptin levels with prediabetes observed in the present study was separate from the effects leptin may have on increasing insulin resistance. Another limitation of the present study was the relatively small size of the recruited cohort, although sample size calculation showed that the study was sufficiently powered to detect significant differences in serum leptin between the two study groups.

In conclusion, and to the best of our knowledge, the present study is the first to have demonstrated a positive link between serum leptin and the risk of prediabetes in a cohort from the MENA region. This association was shown to be independent of the BMI, WC and serum glucose levels. Moreover, the present study is the first to have reported an association between the A allele of rs2167270 and an increased risk of prediabetes using both the univariate and multivariate models.

\section{Acknowledgements}

The authors would like to thank Ms. Khawla Mhedat (Jordan University of Science and Technology) for her technical assistance.

\section{Funding}

The present study was funded by the Deanship of Research at Jordan University of Science and Technology (grant no.541/2018). The article processing charges were partially funded by Deanship of Research at Jordan University of Science and Technology. 


\section{Availability of data and materials}

The datasets generated and/or analyzed during the present study are available from the corresponding author on reasonable request.

\section{Authors' contributions}

MaAA, MuAA and FAM conceived the study. MuAA, MaAA, and MK designed the study. MaAA and LE supervised the study. MK, LE and ZOA contributed to data curation and validated the study. MaAA, MK and RS performed the analysis and experiments. MuAA and MaAA provided the resources and fund acquisition. MK and FAM collected the data. MuAA, MaAA and MK wrote the manuscript. MaAA, ZOA and LE reviewed and edited the manuscript. All authors have read and approved the final manuscript. MK, LE and ZOA confirm the authenticity of all the raw data.

\section{Ethics approval and consent to participate}

All procedures performed in the present study involving human participants were approved by the Jordan University of Science and Technology and King Abdullah University Hospital institutional review board (approval no. 90/118/2018), and in accordance with the 1964 Helsinki Declaration and its later amendments, or comparable ethical standards. Informed consent was obtained from all individual participants included in the study.

\section{Patient consent for publication}

Written informed consent was obtained from all individual participants included in the study.

\section{Competing interests}

The authors declare that they have no competing interests.

\section{References}

1. Meo SA,Usmani AM and Qalbani E: Prevalence of type 2 diabetes in the Arab world: impact of GDP and energy consumption. Eur Rev Med Pharmacol Sci 21: 1303-1312, 2017.

2. Diagnosis and classification of diabetes mellitus. Diabetes Care 37 (Suppl. 1): S81-S90, 2014.

3. Cerf ME: Beta cell dysfunction and insulin resistance. Front Endocrinol 4: 37, 2013.

4. World Health Organization: Diabetes, 2018. https://www.who. int/health-topics/diabetes\#tab=tab_1.

5. International Diabetes Federation: IDF Diabetes Atlas. 9th edition. Brussels, Belgium, 2019. https://www.diabetesatlas. org/en/sections/demographic-and-geographic-outline.html.

6. Abuyassin B and Laher I: Diabetes epidemic sweeping the Arab world. World J Diabetes 7: 165-174, 2016.

7. Ajlouni K, Khader YS, Batieha A, Ajlouni $\mathrm{H}$ and El-Khateeb M An increase in prevalence of diabetes mellitus in Jordan over 10 years. J Diabetes Complications 22: 317-324, 2008.

8. Ajlouni K, Batieha A, Jaddou H,Khader Y, Abdo N,El-KhateebM, Hyassat D and Al-Louzi D: Time trends in diabetes mellitus in Jordan between 1994 and 2017. Diabet Med 36: 1176-1182, 2019.

9. Tabák AG, Herder C, Rathmann W, Brunner EJ and Kivimäki M: Prediabetes: A high-risk state for diabetes development. Lancet 379: 2279-2290, 2012.

10. Nathan DM, Davidson MB, DeFronzo RA, Heine RJ, Henry RR, Pratley R and Zinman B; American Diabetes Association: Impaired fasting glucose and impaired glucose tolerance: implications for care. Diabetes Care 30: 753-759, 2007.
11. Li G, Zhang P, Wang J, Gregg EW, Yang W, Gong Q, Li H, Li H, Jiang Y, An Y, et al: The long-term effect of lifestyle interventions to prevent diabetes in the China Da Qing Diabetes Prevention Study: A 20-year follow-up study. Lancet 371: 1783-1789, 2008.

12. Lindström J, Louheranta A, Mannelin M, Rastas M, Salminen V, Eriksson J, Uusitupa M and Tuomilehto J; Finnish Diabetes Prevention Study Group: The Finnish Diabetes Prevention Study (DPS): Lifestyle intervention and 3-year results on diet and physical activity. Diabetes Care 26: 3230-3236, 2003.

13. Liberopoulos EN, Tsouli S, Mikhailidis DP and Elisaf MS: Preventing type 2 diabetes in high risk patients: An overview of lifestyle and pharmacological measures. Curr Drug Targets 7: 211-228, 2006

14. Skurk T, Alberti-Huber C, Herder C and Hauner H: Relationship between adipocyte size and adipokine expression and secretion. J Clin Endocrinol Metab 92: 1023-1033, 2007.

15. Denroche HC, Huynh FK and Kieffer TJ: The role of leptin in glucose homeostasis. J Diabetes Investig 3: 115-129, 2012.

16. Amitani M, Asakawa A, Amitani $\mathrm{H}$ and Inui A: The role of leptin in the control of insulin-glucose axis. Front Neurosci. 7: 51, 2013.

17. Campfield LA, Smith FJ, Guisez Y, Devos R and Burn P: Recombinant mouse OB protein: Evidence for a peripheral signal linking adiposity and central neural networks. Science 269: 546-549, 1995

18. Murea M, Ma L and Freedman BI: Genetic and environmental factors associated with type 2 diabetes and diabetic vascular complications. Rev Diabet Stud 9: 6-22, 2012.

19. Dasgupta S, Salman M, Siddalingaiah LB, Lakshmi GL, Xaviour D and Sreenath J: Genetic variants in leptin: Determinants of obesity and leptin levels in South Indian population. Adipocyte 4: 135-140, 2014.

20. Hijjawi NS, Al-Radaideh AM, Al-Fayomi KI, Nimer NA, Alabadi HA, Al-Zu'bi RM, Agraib LM, Allehdan SS and Tayyem RF: Relationship of serum leptin with some biochemical, anthropometric parameters and abdominal fat volumes as measured by magnetic resonance imaging. Diabetes Metab Syndr 12: 207-213, 2018.

21. Crujeiras AB, Carreira MC, Cabia B, Andrade S, Amil M and Casanueva FF: Leptin resistance in obesity: An epigenetic landscape. Life 40: 57-63, 2015.

22. Al-Daghri NM, Al-Attas OS, Al-Rubeaan K, Mohieldin M, Al-Katari M, Jones AF and Kumar S: Serum leptin and its relation to anthropometric measures of obesity in pre-diabetic Saudis. Cardiovasc Diabetol 6: 18, 2007.

23. Mazahreh TS, Alfaqih M, Saadeh R, Al-Zoubi NA, Hatamleh M, Alqudah A, Aleshawi AJ and Alzoubi A: The effects of laparoscopic sleeve gastrectomy on the parameters of leptin resistance in obesity. Biomolecules 9: E533, 2019.

24. Hossain IA, Shah R, Jebunnesa F, Zinnat R and Ali L: Association of serum leptin with bta cell dysfunction and insulin resistance among different subgroups of prediabetes. J Diabetes Metab 10: 828,2019

25. Becic T, Studenik C and Hoffmann G: Exercise Increases Adiponectin and Reduces Leptin Levels in Prediabetic and Diabetic Individuals: Systematic Review and Meta-Analysis of Randomized Controlled Trials. Med Sci (Basel) 6: 97, 2018.

26. Sharifi F, Yamini M, Esmaeilzadeh A, Mousavinasab N and Shajari Z: Acylated ghrelin and leptin concentrations in patients with type 2 diabetes mellitus, people with prediabetes and first degree relatives of patients with diabetes, a comparative study. J Diabetes Metab Disord 12: 51, 2013.

27. Hinuy HM, Hirata MH, Sampaio MF, Armaganijan D, Arazi SS, Salazar LA and Hirata RDC: Relationship between variants of the leptin gene and obesity and metabolic biomarkers in Brazilian individuals. Arq Bras Endocrinol Metab 54: 282-288, 2010.

28. Jiang Y, Wilk JB, Borecki I, Williamson S, DeStefano AL, Xu G, Liu J, Ellison RC, Province M and Myers RH: Common variants in the 5 ' region of the leptin gene are associated with body mass index in men from the National Heart, Lung, and Blood Institute Family Heart Study. Am J Hum Genet 75: 220-230, 2004

29. Lakka TA, Rankinen T, Weisnagel SJ, Chagnon YC, Lakka HM, Ukkola O, Boulé N, Rice T, Leon AS, Skinner JS, et al: Leptin and leptin receptor gene polymorphisms and changes in glucose homeostasis in response to regular exercise in nondiabetic individuals: The HERITAGE family study. Diabetes 53: 1603-1608, 2004. 
30. Saadeh N, Alfaqih MA, Mansour H, Khader YS, Saadeh R, Al-Dwairi A and Nusier M: Serum homocysteine is associated with polycystic ovarian syndrome in Jordan. Biomed Rep 9: 439-445, 2018

31. Alfaqih MA, Al-Mughales F, Al-Shboul O, Al Qudah M, Khader YS and Al-Jarrah M: Association of adiponectin and rs1501299 of the ADIPOQ gene with prediabetes in Jordan. Biomolecules 8: E117, 2018.

32. Wauters M, Considine RV, Yudkin JS, Peiffer F, De Leeuw I and Van Gaal LF: Leptin levels in type 2 diabetes: Associations with measures of insulin resistance and insulin secretion. Horm Metab Res 35: 92-96, 2003.

33. Obeidat AA, Ahmad MN, Haddad FH and Azzeh FS: Leptin and uric acid as predictors of metabolic syndrome in Jordanian adults. Nutr Res Pract 10: 411-417, 2016.
34. Mazor R, Friedmann-Morvinski D, Alsaigh T, Kleifeld O, Kistler EB, Rousso-Noori L, Huang C, Li JB, Verma IM and Schmid-Schönbein GW: Cleavage of the leptin receptor by matrix metalloproteinase-2 promotes leptin resistance and obesity in mice. Sci Transl Med 10: eaah6324, 2018.

35. Halford JCG and Blundell JE: Separate systems for serotonin and leptin in appetite control. Ann Med 32: 222-232, 2000.

36. Banihani SA, Elmadhoun RA, Khabour OF and Alzoubi KH: The rs2167270 polymorphism of leptin gene is associated with atopic dermatitis. Dermatoendocrinol 10: e1454191, 2018.

37. Matthews DR, Hosker JP, Rudenski AS, Naylor BA, Treacher DF and Turner RC: Homeostasis model assessment: Insulin resistance and beta-cell function from fasting plasma glucose and insulin concentrations in man. Diabetologia 28: 412-419, 1985. 